\title{
Muscle injections with lidocaine improve resting fatigue and pain in patients with chronic fatigue syndrome
}

This article was published in the following Dove Press journal:

Journal of Pain Research

26 June 2017

Number of times this article has been viewed

\author{
Roland Staud' \\ Taylor Kizer ${ }^{\prime}$ \\ Michael E Robinson² \\ 'Department of Medicine, College \\ of Medicine, ${ }^{2}$ Department of Clinical \\ and Health Psychology, University of \\ Florida, Gainesville, FL, USA
}

Objective: Patients with chronic fatigue syndrome (CFS) complain of long-lasting fatigue and pain which are not relieved by rest and worsened by physical exertion. Previous research has implicated metaboreceptors of muscles to play an important role for chronic fatigue and pain. Therefore, we hypothesized that blocking impulse input from deep tissues with intramuscular lidocaine injections would improve not only the pain but also fatigue of CFS patients.

Methods: In a double-blind, placebo-controlled study, 58 CFS patients received $20 \mathrm{~mL}$ of $1 \%$ lidocaine $(200 \mathrm{mg})$ or normal saline once into both trapezius and gluteal muscles. Study outcomes included clinical fatigue and pain, depression, and anxiety. In addition, mechanical and heat hyperalgesia were assessed and serum levels of lidocaine were obtained after the injections. Results: Fatigue ratings of CFS patients decreased significantly more after lidocaine compared to saline injections $(p=0.03)$. In contrast, muscle injections reduced pain, depression, and anxiety $(p<0.001)$, but these changes were not statistically different between lidocaine and saline $(p>0.05)$. Lidocaine injections increased mechanical pain thresholds of CFS patients ( $p=0.04)$ but did not affect their heat hyperalgesia. Importantly, mood changes or lidocaine serum levels did not significantly predict fatigue reductions.

Conclusion: These results demonstrate that lidocaine injections reduce clinical fatigue of CFS patients significantly more than placebo, suggesting an important role of peripheral tissues for chronic fatigue. Future investigations will be necessary to evaluate the clinical benefits of such interventions.

Keywords: muscle injections, lidocaine, metaboreceptor, chronic fatigue

\section{Introduction}

Chronic fatigue syndrome (CFS) is a disabling disorder whose pathogenesis is only partially understood. It is characterized by unexplained chronic fatigue, exertion intolerance, and sleep and cognitive abnormalities. ${ }^{1}$ It shares many clinical features with fatiguing conditions such as auto-immune illnesses, chronic pain syndromes, multiple sclerosis, heart failure, and cancer. ${ }^{2,3}$ Although chronic fatigue has been reported by patients after infections with herpes viruses (Ebstein-Barr virus, human herpes virus 6 [HHV-6], and cytomegalovirus [CMV]) and Lyme borreliosis, it remains highly controversial whether patients' fatigue is caused or maintained by these infectious agents. ${ }^{4}$ Current treatment approaches for patients with CFS focus almost entirely on symptom relief and in general rely on graded exercise ${ }^{5,6}$ or cognitive behavioral interventions. ${ }^{7}$ Overall, there are no currently accepted CFS treatments directly based on mechanistic understanding of this illness.
Correspondence: Roland Staud of Medicine, University of Florida, 1600 SW Archer Rd, Gainesville,

FL 32610-0221, USA

$\mathrm{Tel}+\mathrm{I} 352273968 \mathrm{I}$

Fax +I 3523928483

Email staudr@ufl.edu 
Chronic fatigue is not only one of the most important symptoms of CFS but also of other medical conditions including congestive heart failure (CHF). ${ }^{8}$ Initially, evaluations of fatigue experienced by such patients have focused on cardiac dysfunction, but measures of heart function only correlate poorly with their clinical fatigue levels. ${ }^{9}$ Accumulating evidence has implicated abnormal concentrations of muscle metabolites, including lactate, $\mathrm{H}+$, and ATP for chronic fatigue, and sensitization of intramuscular afferents as important contributors to fatigue in many chronic illnesses ${ }^{10}$ including CFS. ${ }^{11}$ It has been hypothesized that such afferents become sensitive to metabolic products of skeletal muscle work via metaboreceptors by yet unknown mechanisms. ${ }^{12}$ It is well known that stimulation of metaboreceptors during exercise can result in dyspnea and fatigue - the so-called metabo-reflex ${ }^{10}$ - and early acidosis during exercise appears to be responsible for enhanced metaboreceptors activity in CHF. ${ }^{13}$ While increased metaboreceptors activity may assist in the physiological response to exercise in healthy individuals, excessive stimulation of this reflex may be a source of persistent sympathetic over-activity and reduced vagal activity in patients with chronic fatigue. Such abnormalities are characteristic for CHF, chronic pain, and CFS alike. ${ }^{14-16}$ Thus, metaboreceptors activation may play a key role not only in the onset but also in the progression of illness-associated symptoms in chronic pain, CFS, and CHF by maintaining and stimulating compensatory mechanisms, which are deleterious in the long term. ${ }^{17}$ Overall, abnormal metaboreceptor activity could be relevant for chronic fatigue in many different conditions including CFS, thus providing a new target for research and treatment with focus on peripheral disease mechanisms. ${ }^{18}$ We hypothesized that blockade of peripheral tissue receptors, including metaboreceptor with lidocaine injections, would reduce their input to the central nervous system and thus decrease or abolish fatigue in chronically fatigued patients, including those with CFS. We elected to inject lidocaine into the trapezius and gluteal muscles because of their large size and accessible location.

\section{Methods}

\section{Subjects}

All study procedures were approved by the University of Florida Institutional Review Board prior to the start of subject enrollment and the study protocol conformed to the ethical guidelines of the 1975 Declaration of Helsinki. CFS subjects could not have a history of heart disease, COPD, malignancy, or other systemic disorders including psychiatric illnesses that would be exclusionary for a diagnosis of CFS. ${ }^{19}$ Prior to testing, all subjects underwent a clinical examination and were excluded from the study if they had abnormal findings unrelated to CFS. Use of analgesics, including non-steroidal anti-inflammatory drugs (NSAIDs) and acetaminophen, was not allowed during the study. All subjects were asked to discontinue analgesics for the duration of five drug half-lives before testing, except narcotics that had to be stopped at least 2 weeks prior to study entry. Low-dose muscle relaxants and/ or tricyclic antidepressants ( $\leq 10 \mathrm{mg} /$ day) were permissible during the study for the treatment of insomnia.

\section{Inclusion and exclusion criteria}

Inclusion criteria for participants were, 1) adults over the age of 18 years; 2) the ability to give informed consent; and 3) subjects had to fulfill the 1993 Center for Disease Control Criteria for CFS which stipulate the presence of chronic, unexplained fatigue for $>6$ months that interferes significantly with daily activities or work. In addition, the subjects had to have at least several symptoms, including post-exertional malaise, unrefreshing sleep, impairment of memory or concentration, and muscle/joint pain. Exclusion criteria were, 1) a relevant medical condition besides CFS; 2) current participation in another research protocol that could interfere or influence the outcome measures of this study; 3) the inability to give informed consent; 4) current use of analgesic drugs, hypnotic, or anxiolytic drugs; 5) previous adverse reaction to lidocaine; and 6) previous muscle injections with local anesthetics.

\section{Experimental design}

This study used a parallel group, double-blind, placebocontrolled design. The primary outcomes of this study were change of fatigue and pain. All subjects were trained to rate threshold mechanical and suprathreshold heat pulses to the shoulders, arms, back, and legs. The subjects received experimental pain stimuli immediately before and $30 \mathrm{~min}$ after muscle injections into both shoulders and gluteal muscles. The order of pressure and heat pain stimuli was counterbalanced to prevent order effects. All injections and experimental pain tests were counterbalanced to avoid order effects. Randomization was performed by our investigational pharmacy using mixed block allocation. Study medications were prepared by nursing staff not directly involved in the study and labeled with subject identifiers only. The subjects were lying comfortably on a table during quantitative sensory testing but were asked to sit up during the muscle injections. The interval between heat and pressure stimuli was always $30 \mathrm{~s}$ or until pain aftersensations (AS) were no longer reported by the subjects. 


\section{Ratings of fatigue, pain, and mood}

Ratings of overall fatigue, pain, and mood were obtained before and $30 \mathrm{~min}$ after the muscle injections. A $15 \mathrm{~cm}$ mechanical visual analog scale (mVAS; $0-10)$ was used for ratings of experimental and clinical fatigue and pain. ${ }^{20}$ This scale is anchored on the left by "no fatigue/pain at all" and on the right by "the most intense fatigue/pain imaginable".

The same scale was used for ratings of anxiety/depression. This scale, however, was anchored on the left by "no anxious feelings/depressed feelings at all" and on the right by "most anxious feelings/depressed feelings imaginable".

\section{Muscle injections}

All subjects received one muscle injection into the center of each trapezius muscle, midway between the acromion and the cervical spine, and one injection into the upper medial quadrants of each gluteus maximus muscle using a $27 \mathrm{~g}$ hypodermic needle for a total of four injections. The order of injections was counterbalanced. The injection depth depended on skin thickness but was at least 1 inch to reach muscle tissue. These injection sites were selected because they represented large muscle groups used for activities of daily living. Study medications were prepared in syringes by nurses not involved in the injections. Each syringe used for muscle injections contained either $5 \mathrm{~mL}$ of $1 \%$ lidocaine (50 $\mathrm{mg}$, without additives) or $5 \mathrm{~mL}$ of normal saline. The injections were conducted while the subjects sat on the examination table connected to an electrocardiograph and blood pressure/pulse monitor. The study physician (RS) provided the following instructions for the participants: "the injections you are going to receive contain either a local anesthetic or an inert substance". Then he slowly injected the content of each syringe into the designated muscles over $2 \mathrm{~min}$ in counterbalanced order.

Thirty min and $60 \mathrm{~min}$ after muscle injections, venous blood was drawn for analysis of lidocaine concentrations. The blood samples were immediately sent to the laboratory for analysis.

\section{Expectations of treatment allocation}

Immediately after the muscle injections, the participants were asked by forced choice to indicate whether they believed having received either active study medication or an inert substance.

\section{Experimental pain stimuli}

Experimental heat stimuli were applied before and $\sim 30 \mathrm{~min}$ after the injections to: 1) three marked areas on the volar surface of both forearms $10 \mathrm{~cm}$ proximal of the wrist (areas were separated by $3 \mathrm{~cm}$ ), and 2) the midpoint of the calf $5 \mathrm{~cm}$ lateral to the tibia over the anterior tibialis muscle. These areas were selected because they were distal to the injection sites, thus allowing testing of central nervous system effects of tissue injections on pain sensitivity. For each heat stimulus, the technician placed the thermode on each subject's skin using light pressure while the participant was resting comfortably on the examination table. Similarly, pressure stimuli were applied before and $\sim 30 \mathrm{~min}$ after injections to 1 ) the midpoint of both trapezius muscles (midway between spinal column and acromion), and 2) the medial upper quadrant of the gluteus maximus muscles. These areas were selected because they were identical to the injections sites, thus allowing testing of local nervous system effects of tissue injections on pain sensitivity. Each heat and pressure stimulus was repeated three times in counterbalanced order. The interval between stimuli was always $30 \mathrm{~s}$ or until AS were no longer reported by the subjects. Heat and pressure testing was counterbalanced to avoid order effects.

\section{Heat testing}

\section{Thermal probe}

Precise heat pulses were generated by a Peltier thermode (Pathways; Medoc Advanced Medical Systems, Ramat Yishai, Israel). The heat probe was composed of a Peltier element that provides fast heating rates of up to $10^{\circ} \mathrm{C} / \mathrm{s}$ and cooling rates of up to $8^{\circ} \mathrm{C} / \mathrm{s}$. The thermode provided stimulation of a skin area of $3 \times 3 \mathrm{~cm}$ (surface area: $9 \mathrm{~cm}^{2}$ ). Special hardware and software allowed precise temperature control. The thermal sensors of the thermode were always calibrated before the experiments.

\section{Heat stimuli}

The participants received three $10 \mathrm{~s}$ ramp and hold heat pulses of $44^{\circ} \mathrm{C}, 45^{\circ} \mathrm{C}$, and $46^{\circ} \mathrm{C}$ each to the middle of the volar forearm and the center of the tibialis anterior muscle ("Experimental pain stimuli" section) on both sides. These temperatures were chosen in preliminary experiments, because they were found to be well tolerated by most participants without eliciting withdrawals. The order of testing was counterbalanced. The temperature of the ramp and hold heat pulses increased from $35^{\circ} \mathrm{C}$ to the target temperature over $6 \mathrm{~s}$ and was maintained at the peak temperature for $4 \mathrm{~s}$. The subjects were asked to rate the sensation intensities after each heat pulse using the mVAS. The average of all heat pulse ratings obtained with the same stimulus intensity at each location was used for statistical analyses. 


\section{Mechanical pain threshold testing \\ Dolorimeter}

A calibrated electronic dolorimeter (Wagner Force Measurement, Greenwich, CT, USA) was utilized for $10 \mathrm{~s}$ pressure stimuli. The rubber tip of the dolorimeter was $1 \mathrm{~cm}$ in diameter.

\section{Mechanical stimuli}

For testing of pressure pain thresholds, the electronic dolorimeter was applied to, 1) the center of the shoulders (trapezius muscle), and 2) the upper medial quadrant of the gluteal muscles ("Experimental pain stimuli" section). The order of test locations was counterbalanced to prevent order effects. After the dolorimeter was placed on the target area, pressure was gradually increased by $0.5 \mathrm{~kg} / \mathrm{s}$ until the subject reported pain for the first time. The average of three threshold ratings obtained at the same location was used for statistical analyses.

\section{Data analysis}

Statistical analyses were calculated using SPSS 21.0 software (IBM Corporation, Armonk, NY, USA). Baseline group differences in clinical fatigue, pain, depression, anxiety, and age were analyzed by independent $t$-tests. The effects of muscle injections on experimental fatigue, pain, depression, and anxiety of CFS subjects were analyzed by repeated measures analysis of variance (ANOVA). Significant main and interaction effects were decomposed with appropriate $t$-tests. Linear regressions were performed to assess the contributions of lidocaine blood concentrations to behavioral rating changes. Previous results of lidocaine muscle injections on overall pain in similar study subjects have demonstrated moderate effect size (Cohen's $d=0.7) .{ }^{47}$ Therefore, we used Cohen's power tables estimating that a sample size of 30 subjects would achieve power $>0.8$ with alpha at 0.05 (two-tailed). Significance was set at alpha 0.05.

\section{Results}

\section{Participants}

Prior to enrollment, all subjects provided written informed consent and were told about the nature of the study. All subjects met the 1994 Center for Disease Control Criteria for CFS. A total of 30 CFS subjects (average age [SD] 50.8 [12.4] years) were randomized to lidocaine injections and 28 CFS participants (average age; standard deviation [SD] 50.3 [11.1] years) to saline injections. An independent $t$-test revealed no significant age differences between the groups $(t=0.16$, $p>0.05)$. (see Table 1)

\section{Muscle injections}

All participants tolerated the injections well, except two individuals who demonstrated mild sweating and bradycardia after the injection, which rapidly normalized after they were placed in the supine position. No hematomas, pneumothorax, allergic reactions, or other complications were observed.

\section{Effects of muscle injections on fatigue and pain}

The average (SD) fatigue and pain ratings of CFS subjects assigned to either lidocaine or normal saline are shown in Table 1.

A repeated measures ANOVA was performed to examine the effect of muscle injections on overall fatigue using time (2) as within and condition (2) as between-subjects factors. This analysis demonstrated a significant main effect of time $\left(F(1,43)=20.7 ; p<0.001 ; \eta_{p}^{2}=0.33\right)$ and a significant time $\times$ condition interaction effect $(F(1,43)=5.2 ; p=0.03$; $\left.\eta_{p}^{2}=0.11\right)($ Figure 1).

A similar ANOVA with pain ratings as the dependent variable also showed a significant main effect of time $\left(F(1,43)=26.5 ; p<0.001 ; \eta_{p}^{2}=0.38\right)$ but a non-significant time $\times$ condition interaction $\operatorname{effect}(F(1,43)=0.13 ; p>0.05$; $\left.\eta_{p}^{2}=0.003\right)$ (Figure 2).

Table I Demographics

\begin{tabular}{llll}
\hline & $\begin{array}{l}\text { Lidocaine } \\
\text { injections }\end{array}$ & $\begin{array}{l}\text { Saline } \\
\text { injections }\end{array}$ & p-value \\
\hline Females/males & $28 / 2$ & $26 / 2$ & \\
Age (SD), years & $50.8(12.4)$ & $50.3(11.1)$ & $\mathrm{ns}$ \\
Illness duration (SD), years & $13.9(7.1)$ & $15.4(9.2)$ & $\mathrm{ns}$ \\
\hline
\end{tabular}

Abbreviations: $n s$, not significant; $\mathrm{SD}$, standard deviation.

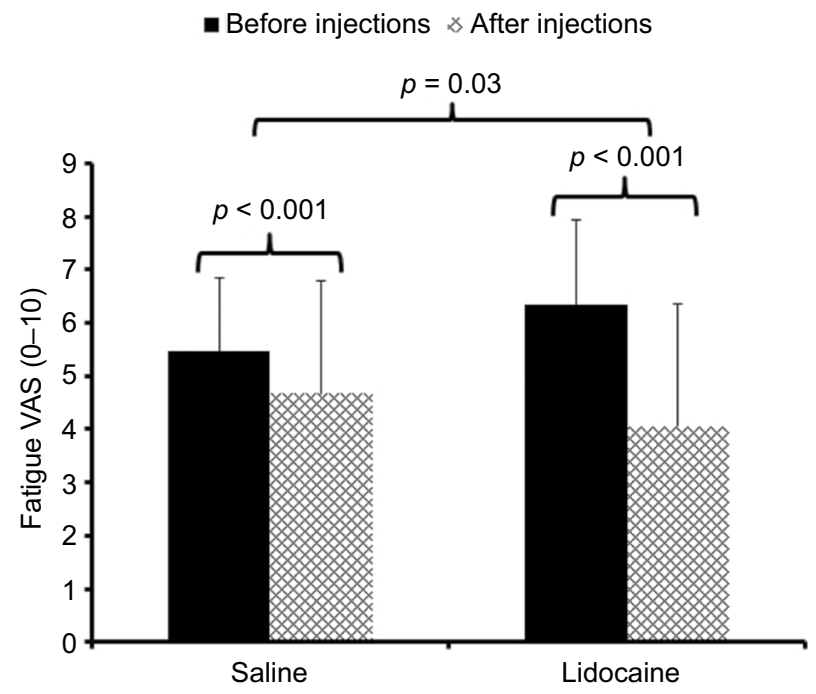

Figure I Mean (SD) ratings of overall fatigue of participants with CFS before (black bars) and after (hatched bars) injections with I\% lidocaine or normal saline.

Notes: All subjects received concomitant injections into both shoulders and buttocks. Fatigue ratings were obtained before and $30 \mathrm{~min}$ after the muscle injections. Fatigue ratings of CFS participants decreased significantly more after lidocaine compared to normal saline injections $(p=0.03)$.

Abbreviations: CFS, chronic fatigue syndrome; $S D$, standard deviation; VAS, visual analog scale. 


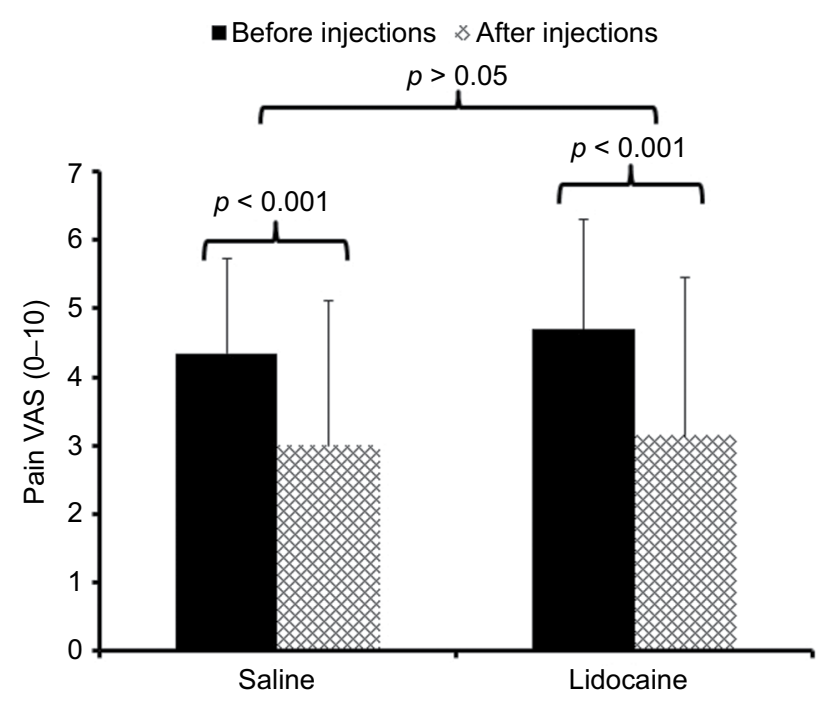

Figure 2 Mean (SD) pain ratings of participants with CFS before (black bars) and after (hatched bars) muscle injections with I\% lidocaine or normal saline.

Notes: All subjects received injections into both shoulders and buttocks in counterbalanced order. Pain ratings were obtained directly before and $30 \mathrm{~min}$ after the muscle injections. Pain ratings of CFS participants decreased significantly over time $(p<0.001)$ but not significantly more after lidocaine compared to normal saline injections $(p>0.05)$.

Abbreviations: CFS, chronic fatigue syndrome; SD, standard deviation; VAS, visual analog scale.

When fatigue and pain ratings were simultaneously introduced into the ANOVA analysis, there was a significant interaction effect of time $\times$ ratings $\times$ condition noted $\left(F(1,43)=5.6 ; p=0.02 ; \eta_{p}^{2}=0.12\right)$. This analysis indicated that lidocaine injections reduced fatigue more effectively than pain without affecting pain ratings more than saline injection.

\section{Effects of muscle injections on depression and anxiety}

The effects of lidocaine or normal saline injections on depression and anxiety are demonstrated in Table 2 . Several repeated measures ANOVAs with condition (2) as independent factors and depression or anxiety ratings as dependent factors were performed. All showed significant main effects of time but non-significant time $\times$ condition interaction effects, indicating that depression and anxiety significantly declined over time but that this effect was not different for lidocaine or saline injections (Table 1).

\section{Effects of muscle injections on mechanical and heat hyperalgesia}

Effects of muscle injections on mechanical hyperalgesia

Mechanical pain thresholds were tested at both shoulders and gluteal muscles before and $30 \mathrm{~min}$ after the injections with either normal saline or lidocaine. After dependent $t$-tests did not show significant differences between ratings at either side, the results of both sides were averaged and entered into repeated measures ANOVAs. The effects of
Table 2 Ratings of fatigue, pain, depression, and anxiety

\begin{tabular}{llllll}
\hline & \multicolumn{2}{l}{ Lidocaine injections } & & \multicolumn{2}{c}{ Saline injections } \\
\cline { 2 - 3 } \cline { 5 - 6 } & Before & After & & Before & After \\
\hline Fatigue VAS (SD) & $6.3(I .6)$ & $4.1(2.3)^{* * * *}$ & & $5.5(1.4)$ & $4.7(2.1)^{* * *}$ \\
Pain VAS (SD) & $4.7(2.1)$ & $3.1(2.0)^{* * *}$ & & $4.3(2.0)$ & $3.0(1.9)^{* * *}$ \\
Depression VAS (SD) & $1.6(2.0)$ & $1.2(1.6)^{* *}$ & & $1.4(1.9)$ & $0.4(0.1)^{* *}$ \\
Anxiety VAS (SD) & $2.4(2.2)$ & $2.0(2.2)^{*}$ & & $2.5(2.5)$ & $1.6(2.2)^{*}$ \\
\hline
\end{tabular}

Notes: $*^{* * *} p<0.001 ; * * p=0.003 ; * p<0.05$.

Abbreviations: SD, standard deviation; VAS, visual analog scale.

muscle injections on mechanical pain thresholds are demonstrated in Figure 3.

Before the injections, the average (SD) mechanical pain threshold at the shoulder was $191.1(100.2) \mathrm{kPa}$ and 231.4 (118.9) $\mathrm{kPa}$ and the mechanical pain threshold at the buttocks was $212.3(119.4) \mathrm{kPa}$ and $292.4(110.1) \mathrm{kPa}$, for subjects randomized to saline and lidocaine, respectively. After the injections, the average (SD) mechanical pain threshold at the shoulders changed to $232.2(112.8) \mathrm{kPa}$ and 315.6 (154.4) $\mathrm{kPa}$ and the mechanical pain threshold at the buttocks changed to $317.4(148.9) \mathrm{kPa}$ and 358.5 (184.0) kPa, for subjects injected with saline and lidocaine, respectively. Repeated measures ANOVAs demonstrated significant effects of time on mechanical thresholds at both locations (all $p<0.001$ ), but time $\times$ condition interactions were only significant at the shoulders $(p=0.03)$ and not at the buttocks $(p>0.05)$ (Table 3$)$, indicating that lidocaine was only more effective than saline in increasing mechanical pain thresholds at the shoulders but not at any other location.

\section{Effects of muscle injections on heat pain ratings}

Heat pain sensitivity was tested with $44^{\circ} \mathrm{C}, 45^{\circ} \mathrm{C}$, and $46^{\circ} \mathrm{C}$ heat pulses at both forearms and legs. Because results obtained with $44^{\circ} \mathrm{C}$ and $46^{\circ} \mathrm{C}$ heat stimuli at the arms and legs were similar to those obtained with $45^{\circ} \mathrm{C}$ stimuli, these findings are not shown here. After dependent $t$-test showed no significant differences between sides, the ratings at both sides were averaged and entered into repeated measures ANOVAs. The effects of tissue injections on heat pain ratings of $45^{\circ} \mathrm{C}$ stimuli at the arms and legs are demonstrated in Figure 4.

Before the injections, the average (SD) ratings of heat pain stimuli at the arms were 3.6 (2.0) VAS units and 4.5 (1.6) VAS units and the ratings of heat pain stimuli at the legs were 3.3 (2.3) VAS units and 4.0 (2.0) VAS units, for subjects randomized to saline and lidocaine, respectively. After the injections, the average (SD) ratings of heat pain stimuli at the arms changed to 3.3 (2.1) VAS units and 4.4 (2.3) VAS units and the ratings of heat pain stimuli at the legs changed to 2.7 (2.2) VAS units and 3.4 (1.8) VAS units, for subjects injected with saline and lidocaine, respectively. 
A Trapezius muscles

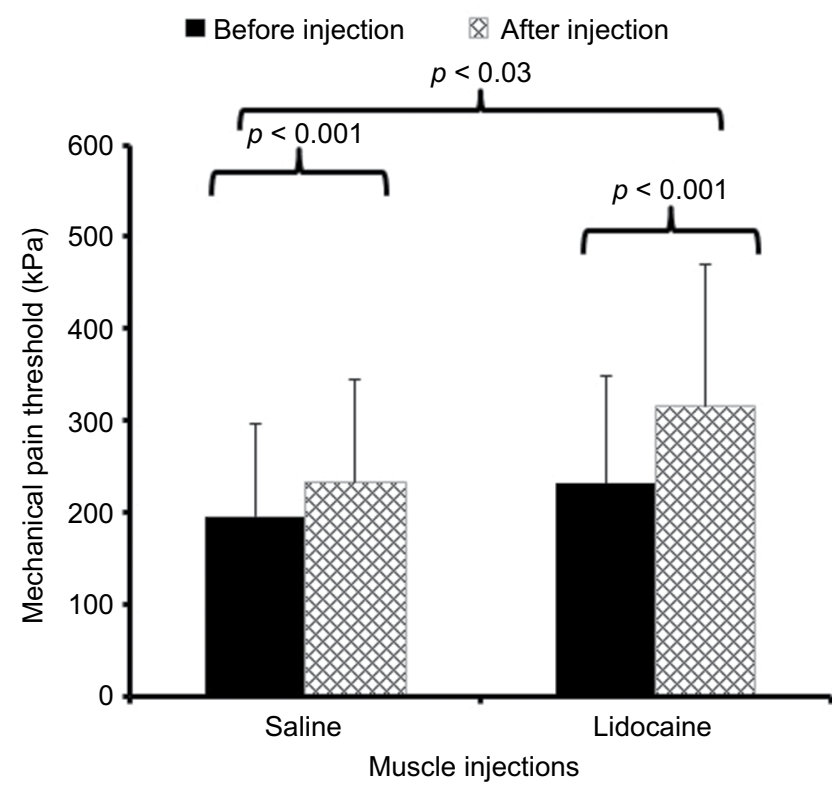

B Gluteal muscles

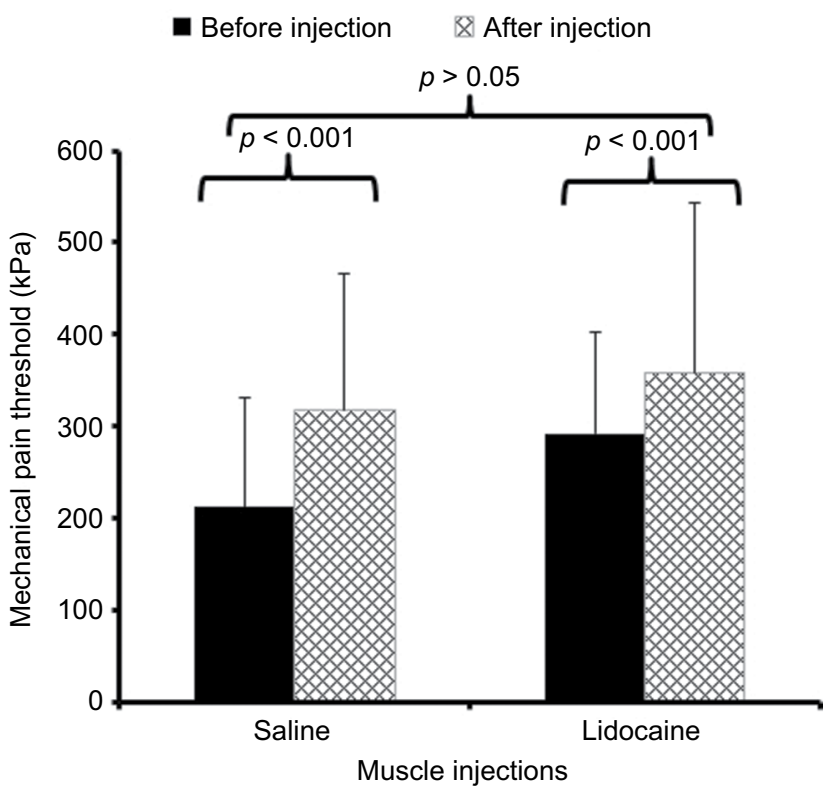

Figure 3 Average pressure pain thresholds (SD) of patients with CFS before (black bar) and 30 min after (hatched bar) muscle injections with I\% lidocaine or normal saline into both shoulder and gluteal areas.

Notes: Pressure pain thresholds were tested at $(\mathbf{A})$ trapezius muscles and $(\mathbf{B})$ gluteal muscles. Pressure pain thresholds significantly increased 30 min after the injections $(p<0.05)$ at all sites, but this effect was only significantly different between lidocaine and normal saline at the shoulders $(p=0.03)$.

Abbreviations: CFS, chronic fatigue syndrome; SD, standard deviation.

Table 3 Time $\times$ condition interaction effects of muscle injections on mechanical pain thresholds

\begin{tabular}{lllllll}
\hline & $\begin{array}{l}\text { Mechanical pain } \\
\text { threshold }(\boldsymbol{F}(\mathbf{I}, \mathbf{4 6}))\end{array}$ & $\eta_{p}^{2}$ & $\boldsymbol{P}$-value & $\begin{array}{l}\text { Time } \times \text { condition } \\
(\boldsymbol{F}(\mathbf{I}, \mathbf{4 6}))\end{array}$ & $\eta_{p}^{2}$ & $\boldsymbol{P}$-value \\
\hline Shoulders & 31.25 & 0.38 & $<\mathbf{0 . 0 0 1}$ & 5.01 & 0.09 & 0.03 \\
Buttocks & 29.52 & 0.38 & $<\mathbf{0 . 0 0 1}$ & $\mathbf{1 . 5 4}$ & 0.03 & $>0.05$ \\
\hline
\end{tabular}

Notes: Bold figures indicate the statistically significant results.

Separate ANOVAs showed small but significant effects of injections on heat pain ratings at the legs (Table 4). All time $\times$ condition interactions, however, were non-significant (all $p>0.05$; Table 3 ). These findings demonstrate that lidocaine injections were not more effective than saline injections for heat pain at both locations.

\section{Lidocaine serum levels after muscle injections}

Mean (SD) lidocaine serum levels obtained from individuals who received this medication were minimal and measured at $1.19(0.35) \mu \mathrm{g} / \mathrm{mL}$ and $1.02(0.27) \mu \mathrm{g} / \mathrm{mL}, 30 \mathrm{~min}$ and $60 \mathrm{~min}$ after the muscle injections, respectively.

\section{Evaluation of systematic biases related to muscle injections}

Effects of muscle injections on behavioral measures

To evaluate the rating changes of study participants after the muscle injections for systematic biases, Pearson's product moment correlations of fatigue, pain, depression, and anxiety were performed. Although fatigue changes were significantly correlated with clinical pain changes $(r=0.65 ; p<0.001)$, there was no significant correlation detected between fatigue, depression, or anxiety change scores (all $p>0.05$ ).

\section{Effects of lidocaine on behavioral measures}

Serum levels of lidocaine were obtained in all subjects $30 \mathrm{~min}$ and $60 \mathrm{~min}$ after the muscle injections ("Lidocaine serum levels after muscle injections" section). However, the use of Pearson's product moment correlation showed no statistically significant relationships between lidocaine serum levels and change scores of fatigue, pain, anxiety, and depression (all $p>0.05$ ).

\section{Treatment allocation concealment}

As problems with treatment allocation concealment can influence the outcome of interventions, all participants were asked (by forced choice) to provide their best guess whether they had received lidocaine or placebo injections. Out of 30 subjects 


\section{A Forearms}

Before injection
B Legs

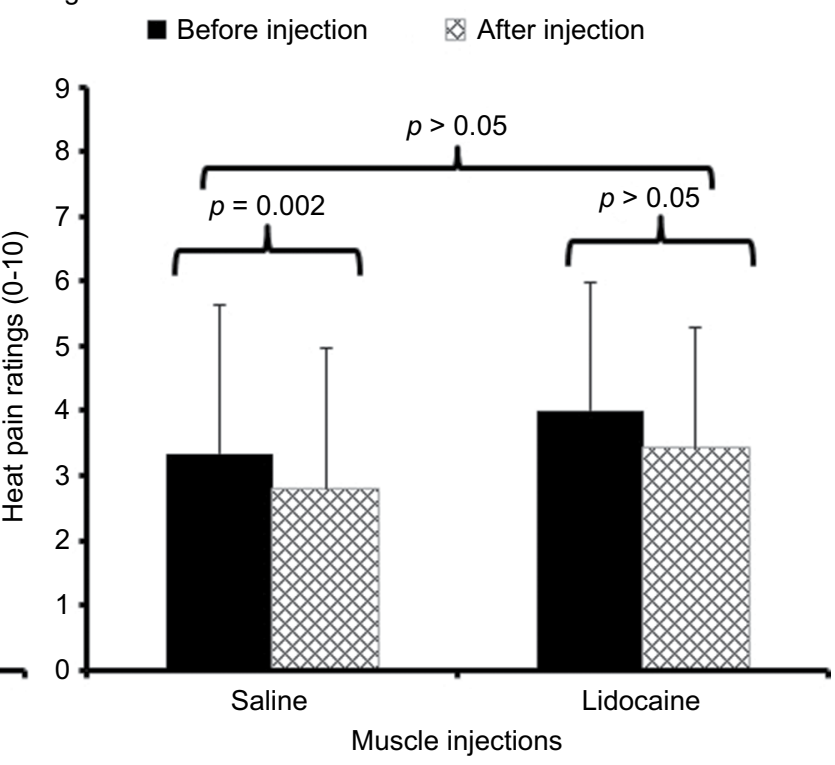

Figure 4 Average heat pain ratings (SD) of $10 \mathrm{~s}$ heat stimuli in patients with CFS at $45^{\circ} \mathrm{C}$ before (black bars) and 30 min after (hatched bars) muscle injections with $1 \%$ lidocaine or normal saline into both shoulder and gluteal areas.

Notes: Supra-threshold heat pain stimuli were applied to the $(\mathbf{A})$ forearms and $(\mathbf{B})$ lower legs (over the anterior tibialis muscles). Heat pain ratings at both sites were not significantly different after injections with lidocaine or normal saline $(p>0.05)$.

Abbreviations: CFS, chronic fatigue syndrome; SD, standard deviation.

Table 4 Time $\times$ condition interaction effects of muscle injections on heat pain ratings

\begin{tabular}{lllllll}
\hline & $\begin{array}{l}\text { Heat pain ratings } \\
(\boldsymbol{F}(\mathbf{I}, \mathbf{5 6}))\end{array}$ & $\eta_{p}^{2}$ & $\boldsymbol{p}$-value & $\begin{array}{l}\text { Ratings } \times \text { condition } \\
(\boldsymbol{F}(\mathbf{I}, \mathbf{5 6}))\end{array}$ & $\eta_{p}^{2}$ & $\boldsymbol{p}$-value \\
\hline Arm $45^{\circ} \mathrm{C}$ & 0.995 & 0.017 & $>0.05$ & 0.267 & 0.005 & $>0.05$ \\
$\operatorname{Leg} 45^{\circ} \mathrm{C}$ & 7.63 & 0.12 & $\mathbf{0 . 0 0 8}$ & 0.0 & 0.0 & $>0.05$ \\
\hline
\end{tabular}

Notes: Bold figure indicates the statistically significant result.

randomized to lidocaine, $24(80 \%)$ declared having received the active study drug and six stated having received placebo. Conversely, out of 28 subjects randomized to saline injections, $21(75 \%)$ thought they had received lidocaine injections and seven believed to have received saline injections. A Fisher's $Z$ test for proportion showed no differences in the proportion of correct guesses between the groups $(z=0.46 ; p=0.65)$.

\section{Discussion}

Previously, a large number of CFS studies have focused on the role of chronic infections/chronic immune activation on chronic fatigue with mixed and often conflicting results. ${ }^{22,23}$ In contrast, very little is known about the contributions of peripheral tissues to chronic fatigue symptoms of CFS patients. In particular, evidence of abnormal concentration of muscle metabolites ${ }^{10}$ and sensitized intramuscular fatigue receptors ${ }^{11}$ has provided the basis for the current hypothesis that fatigue of CFS patients is at least partially affected by peripheral tissue signaling to the central nervous system. This is the first study of CFS patients demonstrating significant reductions of clinical fatigue after muscle injections with $1 \%$ lidocaine. Importantly, the interventions occurred at rest and not after a fatiguing mental or physical task, therefore demonstrating relevant effects on baseline fatigue of CFS patients. Although clinical fatigue of CFS patients was reduced by $38 \%$ after lidocaine injection, which is clinically relevant, some of this effect is most likely related to non-specific factors (expectations, context, etc.). ${ }^{24}$ Moreover, injections with lidocaine did not result in a significantly greater decrease of clinical pain in CFS patients than saline injections. This finding suggests that the observed improvements in fatigue were not mediated by pain reductions. Importantly, CFS patients were unable to reliably distinguish lidocaine from normal saline injections, thus reducing the influence of bias from the results. Overall, the results of this study provide strong support for our hypothesis that peripheral signal transduction to the central nervous system is mechanistically important for the self-reported fatigue of CFS patients at rest. 


\section{Role of peripheral tissues for fatigue}

A large body of evidence supports the important role of peripheral tissue signaling in exercise-related fatigue..$^{25-27}$ Throughout all muscles, free nerve endings from group III and IV muscle afferents are widely distributed that are usually either silent or demonstrate only low background discharge rates $(<1 \mathrm{~Hz})$ at rest. They respond to local mechanical and thermal stimulation and exercise-related muscle metabolites, including lactate, bradykinin, prostaglandins, and potassium ions. ${ }^{28-31}$ Metabolic activity of muscles is sensed by metaboreceptors associated with $\mathrm{A} \delta$ and C-fibers, mediating inputs from alpha- and gammamotor neurons and their presynaptic modulation. The sensations of fatigue can occur during muscle contractions and are often enhanced after exhausting exercise in normal subjects. ${ }^{32}$ Conversely, infusions with acid, ATP, and lactate into muscles can cause fatigue in normal individuals. ${ }^{33}$ However, moderate exercise elicits little long-lasting post-exercise fatigue and no muscle pain in most normal subjects, whereas such symptoms are often exacerbated in CFS patients after mild exercise. ${ }^{34,35}$

The reason for fatigue exacerbations after often minor exercises in CFS patients is only partially understood and seems to be associated with suboptimal central activation of the neuromuscular system. ${ }^{32}$ Previous research by our group has demonstrated evidence for significant contributions of peripheral tissue signaling to chronic fatigue exacerbations, ${ }^{11}$ i.e., when muscle metabolites were allowed to accumulate in the forearms of CFS patients after brief exercise, they reported significantly greater increases of fatigue ratings than during a control condition. ${ }^{11}$ These results suggested not only that activation of metaboreceptors plays an important role for chronic fatigue but also the presence of sensitized fatigue pathways as the same experiments produced less fatigue in healthy controls.

The results of this study support and extend these findings. By blocking peripheral impulse input from deep tissues with lidocaine, fatigue ratings of CFS patients decreased significantly more than with normal saline, again suggesting that peripheral impulse input plays an important role for CFS. Whether the peripheral tissue contributions to fatigue of CFS patients is dependent on particular muscle groups is unclear at this time as all participants received muscle injections into the same four body areas. Future studies will be necessary to demonstrate whether fatigue reductions of CFS patients depend on specific injection sites or lidocaine dose.

\section{Fatigue and pain}

In addition to debilitating fatigue, the majority of patients with CFS experience chronic widespread pain. ${ }^{36,37}$ Conversely, in many conditions with widespread pain, including fibromyalgia syndrome (FM), most patients also report varying levels of fatigue..$^{38}$ Some of the hallmarks of chronic widespread pain are varying degrees of central sensitization. ${ }^{39-41}$ Over the last 10 years, increasing evidence for sensitization of central pain pathways has not only been accumulated in $\mathrm{FM}^{38}$ but also in CFS, ${ }^{36}$ providing mechanistic explanations for increased pain in both disorders. Although no specific tissue abnormalities have been described in many chronic pain disorders including FM, temporomandibular disorder, low back pain, and CFS, it has been proposed that pain and hyperalgesia of such patients may be at least partially dependent on activation of peripheral receptor systems, specifically metaboreceptors. ${ }^{42} \mathrm{We}$ and others have shown that peripheral impulse input appears to be necessary for maintaining central sensitization in many chronic pain conditions including FM. ${ }^{43-47}$

We have previously examined the effects of tissue injections with lidocaine on chronic pain of FM patients. ${ }^{21}$ Similar to this study, four injections into the trapezius and gluteal muscles were performed. Although lidocaine provided significant reductions of local FM pains, there was no superior effect detected for lidocaine on overall pain compared to normal saline injections. ${ }^{21}$ Similarly, no superior effects of lidocaine compared to saline injections on chronic pain of CFS patients was detected in this study.

At this time, we can only speculate why lidocaine injections were only superior in reducing clinical fatigue but not pain of CFS patients. One important difference between peripheral pain and fatigue signaling may be the different magnitude of metaboreceptor and pain receptor contribution for clinical fatigue and pain in CFS, respectively. Whereas large residual nociceptive signaling of muscle groups outside of the injected areas may have prevented significant pain reductions of CFS patients, the metaboreceptor contributions of the injected muscles to fatigue may be substantial enough so that significant improvements of fatigue became detectable. Future lidocaine injection studies with varying numbers of injections sites will be necessary to more conclusively answer these important questions.

\section{Study limitations}

Although unlikely, we cannot exclude a systemic effect of lidocaine injections on chronic fatigue as lidocaine was 
detectable in peripheral blood of CFS patients after the injections. The lidocaine levels in peripheral blood, however, were very low and did not significantly correlate with CFS patients' fatigue reductions.

In general, there were significant effects of muscle injections with either lidocaine or normal saline on fatigue, pain, and mood of CFS patients. This pattern is consistent with placebo effects, ${ }^{48}$ suggesting that non-specific treatment effects occurred with both types of injections; nevertheless, there was a small but significant additive effect from lidocaine noted. Furthermore, it is possible that the lidocaine effect on clinical pain failed to reach significance in our study because of insufficient power.

\section{Conclusion}

The lidocaine-specific effects of muscle injections provide indirect evidence for the dependence of CFS patients' resting fatigue on impulse input from peripheral tissues, likely from metaboreceptors. Muscle injections with lidocaine effectively reduced chronic fatigue by $38 \%$, which may be clinically relevant. The significant correlation between fatigue and pain reductions after lidocaine injections suggests the presence of similar afferent pathways for nociception and fatigue in CFS patients. Future studies will be necessary to identify which peripheral tissues play the most important role for chronic fatigue and whether pharmacological therapies can successfully reduce peripheral tissue contributions to CFS symptoms over extended periods of time.

\section{Acknowledgments}

This study was supported by NIH grant R01 NR014049 and NIH/NCATS Clinical and Translational Science grants UL1 TR000064.The expert assistance of Ricky Madhavan and Meriem Mokhtech is gratefully acknowledged.

\section{Disclosure}

The authors report no conflicts of interest in this work.

\section{References}

1. IOM. Beyond Myalgic Encephalomyelitis/Chronic Fatigue Syndrome: Redefining an Illness. Washington, DC: The National Academic Press; 2015:1-272.

2. Packer TL, Sauriol A, Brouwer B. Fatigue secondary to chronic illness: postpolio syndrome, chronic fatigue syndrome, and multiple sclerosis. Arch Phys Med Rehabil. 1994;75(10):1122-1126.

3. Pepper CM, Krupp LB, Friedberg F, Doscher C, Coyle PK. A comparison of neuropsychiatric characteristics in chronic fatigue syndrome, multiple sclerosis, and major depression. J Neuropsychiatry Clin Neurosci. 1993;5(2):200-205.

4. Wyller VB. The chronic fatigue syndrome - an update. Acta Neurol Scand. 2007;115:7-14.
5. Wallman KE, Morton AR, Goodman C, Grove R, Guilfoyle AM. Randomised controlled trial of graded exercise in chronic fatigue syndrome. Med J Aust. 2004;180(9):444-448.

6. Goudsmit EM, Nijs J, Jason LA, Wallman KE. Pacing as a strategy to improve energy management in myalgic encephalomyelitis/ chronic fatigue syndrome: a consensus document. Disabil Rehabil. 2012;34(13):1140-1147.

7. Prins JB, Bleijenberg G, Bazelmans E, et al. Cognitive behaviour therapy for chronic fatigue syndrome: a multicentre randomised controlled trial. Lancet. 2001;357(9259):841-847.

8. Witte KK, Clark AL. Why does chronic heart failure cause breathlessness and fatigue? Prog Cardiovasc Dis. 2007;49(5):366-384.

9. Falk K, Patel H, Swedberg K, Ekman I. Fatigue in patients with chronic heart failure - a burden associated with emotional and symptom distress. Eur J Cardiovasc Nurs. 2009;8(2):91-96.

10. Piepoli M, Clark AL, Volterrani M, Adamopoulos S, Sleight P, Coats AJ. Contribution of muscle afferents to the hemodynamic, autonomic, and ventilatory responses to exercise in patients with chronic heart failure: effects of physical training. Circulation. 1996;93(5): 940-952.

11. Staud R, Mokthech M, Price DD, Robinson ME. Evidence for sensitized fatigue pathways in patients with chronic fatigue syndrome. Pain. 2015;156(4):750-759.

12. Light AR, Vierck CJ, Light KC, et al. Myalgia and fatigue: translation from mouse sensory neurons to fibromyalgia and chronic fatigue syndromes severity of symptom flare after moderate exercise is linked to cytokine activity in chronic fatigue syndrome. Psychophysiology. 2010;47:615-624.

13. Adamopoulos S, Coats AJ, Brunotte F, et al. Physical training improves skeletal muscle metabolism in patients with chronic heart failure. $J \mathrm{Am}$ Coll Cardiol. 1993;21(5):1101-1106.

14. Newton JL, Okonkwo O, Sutcliffe K, Seth A, Shin J, Jones DE. Symptoms of autonomic dysfunction in chronic fatigue syndrome. QJM. 2007;100(8):519-526.

15. Jones DE, Hollingsworth KG, Taylor R, Blamire AM, Newton JL. Abnormalities in $\mathrm{pH}$ handling by peripheral muscle and potential regulation by the autonomic nervous system in chronic fatigue syndrome. J Intern Med. 2010;267(4):394-401.

16. Martínez-Martínez LA, Mora T, Vargas A, Fuentes-Iniestra M, Martínez-Lavín M. Sympathetic nervous system dysfunction in fibromyalgia, chronic fatigue syndrome, irritable bowel syndrome, and interstitial cystitis: a review of case-control studies. J Clin Rheumatol. 2014;20(3):146-150.

17. Piepoli M, Ponikowski P, Clark AL, et al. A neural link to explain the "muscle hypothesis" of exercise intolerance in chronic heart failure. Am Heart J. 1999;137(6):1050-1056.

18. Okita K, Yonezawa K, Nishijima H, et al. Skeletal muscle metabolism limits exercise capacity in patients with chronic heart failure. Circulation. 1998;98(18):1886-1891.

19. Reeves WC, Lloyd A, Vernon SD, et al. Identification of ambiguities in the 1994 chronic fatigue syndrome research case definition and recommendations for resolution. BMC Health Serv Res. 2003;3(1):25.

20. Price DD, Bush FM, Long S, Harkins SW. A comparison of pain measurement characteristics of mechanical visual analogue and simple numerical rating scales. Pain. 1994;56(2):217-226.

21. Staud R, Weyl EE, Bartley E, Price DD, Robinson ME. Analgesic and anti-hyperalgesic effects of muscle injections with lidocaine or saline in patients with fibromyalgia syndrome. Eur J Pain. 2014;18(6): 803-812.

22. Blundell S, Ray KK, Buckland M, White PD. Chronic fatigue syndrome and circulating cytokines: A systematic review. Brain Behav Immun. 2015;50:186-195.

23. Twisk FN. A critical analysis of the proposal of the Institute of Medicine to replace myalgic encephalomyelitis and chronic fatigue syndrome by a new diagnostic entity called systemic exertion intolerance disease. Curr Med Res Opin. 2015;31(7):1333-1347. 
24. Benedetti F, Amanzio M. Mechanisms of the placebo response. Pulm Pharmacol Ther. 2013;26(5):520-523.

25. Gandevia SC. Spinal and supraspinal factors in human muscle fatigue. Physiol Rev. 2001;81(4):1725-1789.

26. Martin PG, Smith JL, Butler JE, Gandevia SC, Taylor JL. Fatiguesensitive afferents inhibit extensor but not flexor motoneurons in humans. J Neurosci. 2006;26(18):4796-4802.

27. Taylor JL, Todd G, Gandevia SC. Evidence for a supraspinal contribution to human muscle fatigue. Clin Exp Pharmacol Physiol. 2006;33(4):400-405.

28. Franz M, Mense S. Muscle receptors with group IV afferent fibres responding to application of bradykinin. Brain Res. 1975;92(3):369-383.

29. Fock S, Mense S. Excitatory effects of 5-hydroxytryptamine, histamine and potassium ions on muscular group IV afferent units: a comparison with bradykinin. Brain Res. 1976;105(3):459-469.

30. Mense S. Sensitization of group IV muscle receptors to bradykinin by 5-hydroxytryptamine and prostaglandin E2. Brain Res. 1981;225(1):95-105.

31. Graven-Nielsen T, Mense S. The peripheral apparatus of muscle pain: evidence from animal and human studies. Clin J Pain. 2001;17(1): $2-10$.

32. Zwarts MJ, Bleijenberg G, van Engelen BGM. Clinical neurophysiology of fatigue. Neurophysiol Clin. 2008;119(1):2-10.

33. Pollak KA, Swenson JD, Vanhaitsma TA, et al. Exogenously applied muscle metabolites synergistically evoke sensations of muscle fatigue and pain in human subjects. Exp Physiol. 2014;99(2):368-380.

34. VanNess JM, Snell CR, Stevens SR. Diminished cardiopulmonary capacity during post-exertional malaise. J Chronic Fatigue Syndr. 2007;14: $77-85$.

35. VanNess JM, Stevens SR, Bateman L, Stiles TL, Snell CR. Postexertional malaise in women with chronic fatigue syndrome. $J$ Womens Health. 2010;19(2):239-244.

36. Meeus M, Nijs J. Central sensitization: a biopsychosocial explanation for chronic widespread pain in patients with fibromyalgia and chronic fatigue syndrome. Clin Rheumatol. 2007;26(4):465-473.
37. Buchwald D. Fibromyalgia and chronic fatigue syndrome: similarities and differences. Rheum Dis Clin North Am. 1996;22(2):219-243.

38. Staud R. Cytokine and immune system abnormalities in fibromyalgia and other central sensitivity syndromes. Curr Rheumatol Rev. 2015;11(2): $109-115$.

39. Staud R. Chronic widespread pain syndrome and fibromyalgia: Two sides of the same coin? Curr Rheumatol Rep. 2009;11(6):433-436.

40. Staud R. Is it all central sensitization? Role of peripheral tissue nociception in chronic musculskeletal pain. Curr Rheumatol Rep. 2010;12(6): 448-454.

41. Staud R. The important role of CNS facilitation and inhibition for chronic pain. Int J Clin Rheumtol. 2013;8(6):639-646.

42. Vierck CJ Jr. Mechanisms underlying development of spatially distributed chronic pain (fibromyalgia). Pain. 2006;124(3):242-263.

43. Staud R, Nagel S, Robinson ME, et al. Enhanced central pain processing of fibromyalgia patients is maintained by muscle afferent input: a randomized, double-blind, placebo controlled trial. Pain. 2009;145(1-2): 96-104.

44. Affaitati G, Costantini R, Fabrizio A, Lapenna D, Tafuri E, Giamberardino MA. Effects of treatment of peripheral pain generators in fibromyalgia patients. Eur J Pain. 2011;15(1):61-69.

45. Alonso-Blanco C, Fernández-de-las-Peñas C, Morales-Cabezas M, Zarco-Moreno P, Ge HY, Florez-García M. Multiple active myofascial trigger points reproduce the overall spontaneous pain pattern in women with fibromyalgia and are related to widespread mechanical hypersensitivity. Clin J Pain. 2011;27(5):405-413.

46. Zhou QQ, Verne GN. New insights into visceral hypersensitivityclinical implications in IBS. Nat Rev Gastroenterol Hepatol. 2011;8(6): 349-355.

47. Kosek E, Ordeberg G. Abnormalities of somatosensory perception in patients with painful osteoarthritis normalize following successful treatment. Eur J Pain. 2000;4(3):229-238.

48. Wager TD, Atlas LY. The neuroscience of placebo effects: connecting context, learning and health. Nat Rev Neurosci. 2015;16(7):403-418.
Journal of Pain Research

\section{Publish your work in this journal}

The Journal of Pain Research is an international, peer reviewed, open access, online journal that welcomes laboratory and clinical findings in the fields of pain research and the prevention and management of pain. Original research, reviews, symposium reports, hypothesis formation and commentaries are all considered for publication.

\section{Dovepress}

The manuscript management system is completely online and includes a very quick and fair peer-review system, which is all easy to use. Visit http://www.dovepress.com/testimonials.php to read real quotes from published authors. 